\title{
Une paraphrase poétique de l'Ave Maria dans l'œuvre de Jean Miélot. Présentation, commentaire, édition
}

\section{Gérard Gros}

\section{(2) OpenEdition}

Journals

\section{Édition électronique}

URL : https://journals.openedition.org/studifrancesi/4539

DOI : 10.4000/studifrancesi.4539

ISSN : 2421-5856

Éditeur

Rosenberg \& Sellier

\section{Édition imprimée}

Date de publication : 1 avril 2012

Pagination : 59-65

ISSN : 0039-2944

\section{Référence électronique}

Gérard Gros, « Une paraphrase poétique de l'Ave Maria dans l'œuvre de Jean Miélot. Présentation, commentaire, édition », Studi Francesi [En ligne], 166 (I | LVI) | 2012, mis en ligne le 30 novembre 2015, consulté le 18 novembre 2021. URL : http://journals.openedition.org/studifrancesi/4539 ; DOI : https://doi.org/10.4000/studifrancesi.4539

\section{(c) (i) (2)}

Studi Francesi è distribuita con Licenza Creative Commons Attribuzione - Non commerciale - Non opere derivate 4.0 Internazionale. 


\section{Une paraphrase poétique de l'Ave Maria dans l'œuvre de Jean Miélot. Présentation, commentaire, édition}

Le manuscrit de Paris, BnF, fr. 9198, est le premier de deux volumes ${ }^{1}$ contenant des œuvres en prose française, par Jean Miélot ${ }^{2}$. Le titre pourrait en être Vie et Miracles de Nostre Dame 3 . Bien connu pour ses nombreuses miniatures en camaïeu, cet exemplaire atteste, justement par l'image, à la fois la destination ducale de l'ouvrage - à Philippe le Bon - et le rôle de l'auteur-écrivain ${ }^{4}$.

À la fin de ce premier volume, au fol. $151^{v 5}$, placée en tête de page, une souscription indique:

Cy fine le Livre de la Vie et Miracles de Nostre Dame / mere de Jhesus, qui fu finit a Le Haye en Hollande / le v jour du mois d'avril. L'an de Nostre Seigneur / Mil quatrecens cinquante six. ${ }^{6}$

Dans ce premier volume (fr. 9198), on peut lire, insérés dans la Vie de la Vierge, fol. $17^{\mathrm{r}}-19^{\mathrm{r}}$, les textes de deux poèmes à forme fixe $\mathrm{e}^{7}$. Il n'est pas indifférent de considérer la place (et la raison d'être) de ces deux pièces dans le livre ${ }^{8}$, et d'envisager à présent, quant à sa nature et sa destination, la première.

(1) Le second est coté Paris, BnF, fr. 9199. Léopold Delisle a toutefois démontré que ce second volume est en fait une copie - servile - du manuscrit d'Oxford, Bodleian Library, Douce 374, lui-même publié en Angleterre en 1885 (Miracles de Nostre Dame, collected by Jean Mielot, secretary to Philip the Good, duke of Burgundy. Reproduced in fac simile from Douce manuscript 374 in the Bodleian library for John Malcom of Poltalloch, with Text, Introduction, and Annotated Analysis by George F. Warner, M.A. Westminster, Nichols and Sons, 1885, Collection du Roxburghe Club). Voir L. DELISLE, Les Miracles de Notre-Dame. Rédaction en prose de Jean Miélot, «Bulletin historique et philologique du Comité des travaux historiques et scientifiques» (Ministère de l'Instruction publique, des Beaux-Arts et des Cultes), 1886, 1-2, pp. 32-45, et spécialement pp. 40-41: «notre manuscrit 9198 est le premier tome de l'exemplaire dont le manuscrit bodléien est le tome second» (p. 41). Ce second tome contient 74 miracles.

(2) Voir Bibliothèque Nationale. Catalogue général des manuscrits français par H. OMONT. Ancien supplément français. I, $\mathrm{N}^{\text {os }} 6171-9560$ du fonds français, Paris, Ernest Leroux, 1895, pp. 323-324. La cote ancienne de ces deux volumes manuscrits était: Supplément français 3007, 2-3.

(3) Voir, infra, la première des citations.

(4) Voir L. DelisLE, art. cité, p. 35: «Le manuscrit est orné de 58 peintures en camaïeu, dont les deux plus remarquables sont celles des folios 1 et 19 . Philippe le Bon, à genoux, est présenté par son patron et par saint André à la sainte Vierge, qui tient l'enfant Jésus et qui est assise près de sainte Anne. Dans le haut du tableau, les armoiries du duc sont soutenues par un ange; au-dessus, la devise Aultre n'aray. Au folio 19, on voit Jean Miélot dans son étude, au milieu des livres, écrivant sur une feuille de parchemin; il faut remarquer les besicles posées sur un des meubles qui garnissent l'étude».

(5) Le fol. 152 est resté blanc.

(6) Pour le mode d'édition de ce texte (et des suivants), voir infra, n. 46.

(7) Pour l'étude et l'édition du second de ces poèmes, voir G. Gros, 'Lit preparé au fil du Roy des rois', chant royal amiénois de l'année 1448: un texte retrouvé, à paraître dans les Comptes rendus de l'Académie des Inscriptions et Belles-Lettres (année 2011).

(8) Pour une édition récente de ces deux chants royaux, voir 'La Vie et miracles de Nostre Dame' de Jean Miélot. A Partial Critical Edition, by J. ReuNING. Chapel Hill, 2003, pp. 41-47. Cet ouvrage publie précisément les fol. $\mathrm{A}^{\mathrm{r}}-49^{\mathrm{r}}$ du manuscrit de Paris, BnF, fr. 9198, soit (avec les différents textes en prose) 10 des 41 Miracles contenus dans ce premier volume. 
On trouvera donc ci-dessous, pour commencer, un examen, sur nouveaux frais, de la disposition des textes autour des deux chants royaux, puis un commentaire et, pour finir, l'édition du poème sur l'Ave Maria.

\section{La place des deux chants royaux}

C'est en vain qu'on chercherait l'annonce de ces deux chants royaux dans la Table liminaire au premier volume (fol. $\mathrm{A}^{\mathrm{r}}$ ), dont voici précisément le texte pour cette partie de l'œuvre:

$S^{\prime}$ ensieuvent les rubriques ${ }^{10}$ de la table de la Vie / et Miracles de Nostre Dame. Et primes ${ }^{11}$

La $a^{12}$ genealogie de Nostre Dame / mere de Dieu.

Prologue de saint Jherome sur la vie de la / tres bieneuree Vierge Marie, qu'il composa I a la requeste d'un sien disciple en la fourme et ma/niere qui s'ensieut.

La Vie de la glorieuse Vierge Marie mere de / Nostre Sauveur Jhesucrist.

Ung petit traictiet de l'Assumption de la tres / glorieuse Vierge Marie ${ }^{13}$.

Les deux chants royaux se trouvent placés entre Vie et Assomption, donc entre l'avant-dernier et le dernier des textes annoncés ci-dessus. Mais ici encore, il convient de préciser, puisque la Table liminaire est par trop succincte.

D'abord figure, au fol. $17^{\mathrm{r}}$, une sorte de rubrique conclusive, ou, si l'on veut, de souscription (copiée, après une lettrine, à l'encre brune):

Cy fine comment la Vierge Marie fu conceue. / Comment elle fu nee. Comment elle fu I nourrie dedens le Temple. Comment elle fu I mariee a Joseph. Comment elle conçut par I l'Anunciation de l'angele. Comment elle enfanta / et s'en fuy ${ }^{14}$.

La Vie aura donc commencé à la conception de la Vierge, et le récit s'en achève (provisoirement?) après la Nativité de Jésus, à la Fuite en Égypte.

Vient ensuite une enluminure de l'Annonciation.

Puis une nouvelle rubrique - au sens propre (elle est écrite à l'encre rouge) - sert cette fois d'annonce:

Cy commencent deux chantz royaulx baladez en l'onneur / et reverence de la benoite Vierge Marie, mere de Dieu.

Ici prennent donc place les deux poèmes à forme fixe.

Une seconde enluminure, au fol. $19^{\mathrm{r}}$, suit le texte du second chant royal; c'est

(9) Cette première phrase est rubriquée au sens propre: écrite en rouge.

(10) = "titres".

(11) = "premièrement".

(12) Au commencement de cette phrase et des suivantes est dessinée une lettrine, or sur fond bleu et rouge.

(13) Ensuite, après un blanc d'une ligne, est inscrit en rouge le titre Rubriques des miracles; s'alignent en effet au-dessous, commençant par une lettrine, les titres des Miracles. Ainsi, dans l'ordre: Miracle de la repentanche de Theophilus qui / renya
Jhesucrist et puis desservy avoir pardon / de la Vierge Marie. Il bailla au diable d'enfer cy/rographe signee de son anel. / Miracle d'un archevesque de Thoulecte nommé / Hildefons et de son successeur appellé Siacgre. / Miracle d'un religieux moyne qui estoit secreltain en son couvent emprés lequel il se noya et I puis revesqui par les merites de Nostre Dame. On constate que les deux premiers textes obéissent à la disposition qu'ils ont - en position initiale - dans l'œuvre de Gautier de Coinci.

(14) Sur le mode de transcription de ce texte, voir derechef infra, n. 46. 
la célèbre image montrant Jean Miélot, dans son cabinet de travail et devant son pupitre, occupé à copier ${ }^{15}$.

Puis, en rouge:

S'ensieut ung petit prologue sur l'Assumption de la Vierge / Marie, translaté de latin en françois par Jo. Milot ${ }^{16}$.

Cette seconde enluminure précédant la rubrique appelle la suite de l'ouvrage, l'autre versant apocryphe du récit de la Vie de la Vierge: sur sa fin terrestre. Or ce qui est ici annoncé échappe encore à la Table liminaire, puisqu'il s'agit d'un «petit» prologue au récit de l'Assomption. La copie de ce prologue, petit prologue, en effet, prend fin au fol. $20^{\mathrm{r}}$, après une double enluminure ( $C y$ fine le prologue de ce livret). Sur la même page est annoncé, par une phrase écrite en rouge, le dernier texte portant sur la Vie de la Vierge:

\section{Cy commence ung petit traictié de l'Assumption Nostre Dame.}

Il s'agit du dernier traité enregistré avant les Miracles dans la Table liminaire.

Pour résumer de façon simple: la rubrique concluant la Vie de la Vierge se trouve au sommet du fol. $17^{\mathrm{r}}$, c'est-à-dire après deux cahiers de parchemin comportant chacun 8 feuillets $^{17}$. Tout se passe comme si, la Table liminaire une fois établie, et après la copie de la Vie de la Vierge (jusqu'à la fin de la Fuite en Égypte), on avait ajouté, sur un nouveau cahier de parchemin, du texte qu'il n'avait pas été prévu dès le départ d'incorporer à ce premier volume.

\section{La présentation du chant royal}

Il est heureux que la rubrique annonçant, au fol. $17^{\mathrm{r}}$, les deux textes $-C y$ commencent deux chantz royaulx baladez en l'onneur / et reverence de la benoite Vierge Marie, mere de Dieu - insiste sur les caractéristiques formelles de poèmes à gabarit fixe - chantz royaulx baladez -, car, dans le manuscrit, la transcription des deux textes est à longues lignes, et, si l'on peut dire, de plus en plus prosaïque. Le premier, «Ave fu dit...» commence par une grande lettrine; on repère encore cinq lettrines de moindre dimension, or sur fond bleu et rouge, pour les initiales des débuts de couplets suivants $^{18}$. Si le présent texte est copié à longues lignes, il comporte un point après le libellé de chaque vers, et la majuscule marque le début du vers suivant. Un alinéa est ménagé après le texte de chaque strophe. Mais il n'y a pas d'alinéa entre la fin de ce poème et le commencement du second ${ }^{19}$.

On constate que la première, la deuxième et la cinquième strophes occupent neuf lignes, la sixième (celle de l'envoi) cinq, mais la troisième huit lignes, et la quatrième onze. La disparité dans les proportions des couplets s'éclaire à la lecture du

(15) Voir, ci-dessus, n. 4, la description qu'en donne L. Delisle. Une excellente reproduction de cette image, sertie de texte, est publiée dans Histoire des bibliothèques françaises, Les bibliothèques médiévales du VI siècle à 1530, sous la direction d'A. Vernet, Paris, Promodis, Éditions du Cercle de la Librairie, 1989 , p. 254; c'est la planche 16 de l'article de G. HASENOHR, L'essor des bibliothèques privées aux XIV et XV siècles, pp. 214-263 de l'ouvrage.

(16) Sic pour la graphie du nom. Il est vrai que le narrateur du «petit prologue» s'appelle Miletus.
(17) Voir L. DelisLe, art. cité, p. 34: «Le manuscrit 9198, relié en maroquin rouge au XVIII ${ }^{\mathrm{e}}$ siècle, consiste en 155 feuillets, cotés A-C et 1-152; il est formé de 20 cahiers, dont le premier est consacré à une table préliminaire et dont les 19 autres sont composés chacun de 8 feuillets».

(18) Â la suite, sans alinéa, ni aucune lettrine, ni aucun espace blanc entre un couplet et le suivant, est copié Sunamitis, le second chant royal.

(19) saluee. Sunamitis: ainsi se présente le passage du premier au second chant royal, fol. $18^{\mathrm{r}}$, au commencement de la ligne 18 . 
poème. Il s'agit bien - comme pour le texte copié à la suite et conformément à la rubrique d'annonce - d'un chant royal selon la définition la plus stricte: cinq onzains de décasyllabes sur cinq rimes ${ }^{20}$ et un envoi (quintil pour ce premier texte, quatrain pour le second), naturellement à refrain.

Or c'est une pièce assez connue que ce premier des deux chants royaux, «Ave fu dit pour salutacïon». Jean Sonet l'enregistre dans son Répertoire ${ }^{21}$. Louis Mourin, sept années plus tôt, l'a présentée puis éditée dans «Scriptorium» ${ }^{22}$, d'après le manuscrit de Bruxelles, KBR, 9270, fol. 396 $-397^{\mathrm{r23}}$ : l'unité, dans ce recueil, entre la pièce lyrique et le reste de l'ouvrage, est assurée par le thème de la prière, puisque ce manuscrit publie le Traité sur l'Ave Maria, de Jean Miélot, qu'on date de 1458. Le chant royal - on l'a vu par la souscription que porte le manuscrit de Paris - est en principe antérieur à ce traité d'au moins deux ans. Au moins découvre-t-on que le texte conservé par le manuscrit de Bruxelles n'est pas un unicum, puisque nous pouvons y ajouter cette version du manuscrit de Paris, BnF., fr. 9198, fol. $17^{\mathrm{r}}-18^{\mathrm{r}}$, où le poème, illustrant la fête de l'Annonciation, contribue cette fois à célébrer le rôle de la Vierge Marie dans l'œuvre de la Rédemption.

Mieux: la version du manuscrit de Paris permet d'amender celle du manuscrit de Bruxelles, et par conséquent de parfaire le texte voulu par Miélot. Louis Mourin n'a pas numéroté les vers de son édition du texte. Or, rendant compte de ce travail avec l'acuité de vision qui était la sienne, Arthur Långfors, se fondant sur le gabarit de la strophe, a déduit qu'il manque un vers, le quatrième, au troisième couplet ${ }^{24}$. C'est «Plaisir, liesse et joye infinitive», selon le manuscrit de Paris. Toutefois, dans ce dernier témoin, manque aussi un vers, le huitième, au troisième couplet. C'est bien pourquoi, d'ailleurs, la copie à lignes longues n'excède pas, pour ce couplet, huit lignes. Le manuscrit de Bruxelles permet de combler la lacune, qui est, à suivre sa leçon ${ }^{25}:$ «Ancoires fust sa lignie asservie».

Cependant, le manuscrit de Paris lui-même permettait la rectification, au prix d'un détour. En effet, la copie du quatrième couplet, atteignant onze lignes, comporte (par conséquent, serait-on tenté de dire) deux vers de trop. Ce sont, entre les vers 40 et 41: «La grief paine que Adam ot deservie / Encores fust la lignie asservie»; ces vers, où ils sont insérés, ne donnent pas de sens à la phrase, et font même du propos un non-sens. Cependant le premier peut donner l'impression du déjà lu, puisqu'il s'agit en effet, au troisième couplet, du vers 29 , qui précède immédiatement le vers manquant. Tout se passe comme si, s'étant relu ou ayant été relu, le copiste (Jean Miélot?), constatant son omission, la réparait au couplet suivant, en insérant d'abord le vers déjà lu, comme un signal, afin que la lecture restitue la version correcte en ajoutant à sa suite, et en revenant en arrière, le vers manquant. Toutefois on ne repère aucun signe graphique invitant au renvoi ou à la rectification dans le texte.

(20) Curieusement, ou fortuitement, d'un chant royal à l'autre, le genre des rimes est inversé dans le couplet; celui-ci, dans la première pièce, a pour schéma: ababccddedE (rimes a et c masculines); dans la seconde: $a \mathrm{~b} a \mathrm{~b} c c \mathrm{ddedE}$ (rimes $\mathrm{b}$, d et e masculines).

(21) Répertoire d'incipit de prières en ancien français, Genève, Droz, 1956 (Publications Romanes et Françaises, LIV), 116, p. 22.

(22) Poésies religieuses françaises inconnues, dans des manuscrits de Bruxelles et d'Évora, «Scriptorium», III, 1949 , pp. 218-229, notamment pp. 218 219 (présentation) et pp. 228-229 (édition).
(23) Voir le compte rendu d'A. LÅngFORs, Notes sur quelques manuscrits français de Bruxelles et un livre d'Heures d'Évora (Portugal), «Neuphilologische Mitteilungen», LII, 1951, pp. 158-165, notamment pp. 164-165 (avec des suggestions d'amendements).

(24) Ibid., p. 165: «Le chant royal comprend cinq onzains et un envoi, tous à refrain. Le schéma métrique (10 ababccddedE) permet de constater qu'au couplet III, trop court d'un vers, c'est le quatrième qui manque».

(25) v. 30. 


\section{La paraphrase poétique de l'Ave Maria}

Dieu sait combien l'Ave Maria paraphrasé s'est décliné dans la diversité des formes $^{26}$. La méthode employée par le poète est loin d'être inusitée: c'est dans la citation de chacun des six premiers mots de la prière usuelle, en latin, posés au début du couplet, que la nouveauté du propos prend son essor; du vocable latin naît la phrase française, en toute harmonie. Le sujet, ici comme en tant d'autres pièces de même genre, est moins la glose, en effet, que la paraphrase. Il s'agit moins d'expliquer le texte que de justifier l'étonnement qu'on éprouve à méditer ce qu'il implique: le salut. Même lorsque le poème en français se réfère à Grâce [de] Dieu comme à Sapience pour détailler le projet de la Rédemption, le propos est encore une déclaration de gratitude à Dieu pour la miséricorde infinie qu'il manifeste à la créature humaine. Aussi, par voie de conséquence, en une époque où l'on disposait, suivant la "rhétorique seconde", de formes idoines aux sujets et préparées pour eux, ce poème est une prière de louange et non de pétition. La forme qu'il met en œuvre n'est donc ni le douzain d'Hélinant (isométrique ou de préférence hétérométrique), employé pour la requête, ni, dans la strophe à forme fixe elle-même, le distique - couple de vers à rimes suivies -, employé pour la narration.

Le gabarit du poème «Ave fu dit» rend perplexe. Aucun des 13 articles enregistrés par Édith Brayer, dans son Catalogue $e^{27}$, sous la rubrique Ave Maria ${ }^{28}$, ne se présente sous la forme d'un chant royal ou même, plus simplement, d'une ballade. Il est vrai que l'inventaire ne descend pas plus bas que le XIV siècle. Au Répertoire de Jean Sonet ${ }^{29}$, dans lequel sont référencées 40 paraphrases de l'Ave Maria - 29 anonymes et 11 attribuées, dont 5 à des poètes du $\mathrm{XV}^{\mathrm{e}}$ siècle -, on constate que les formes employées le plus volontiers à la fin du Moyen Âge sont soit le huitain d'octosyllabes ${ }^{30}$, soit le lai (qui reprend le gabarit du douzain d'Hélinant) ${ }^{31}$. Nul espoir de trouver dans cet inventaire un quelconque poème strophique à refrain pour paraphrase de l'Ave Maria.

Dans son supplément ${ }^{32}$, Keith Val Sinclair ajoute 10 entrées sur le sujet - 6 pièces anonymes et 4 d'auteurs connus. Pour parler de la fin du XV siècle (et des années ultérieures), on constate que le Rhétoriqueur, en l'occurrence Guillaume Cretin ${ }^{33}$ - mais tel serait aussi le cas pour un Jean Molinet ${ }^{34}$ - rime son oraison dans un gabarit strophique rare probablement, conçu peut-être comme exceptionnel, une variante par amplification du douzain d'Hélinant.

Jusqu'à plus ample informé, cet Ave Maria en chant royal, enregistré dès Sonet, comme on l'a vu, dans son Répertoire ${ }^{35}$, est au point de vue formel un unicum. Du point de vue formel, Louis Mourin notait qu'il n'est pas conforme au gabarit

(26) Voir par exemple G. Gros, 'Ave, Vierge Marie...' - Etude sur les prières mariales en vers français (XII $-X V^{e}$ siècles), Presses Universitaires de Lyon (Collection XI-XVII Littérature, 8), 2004, pp. 109 150.

(27) «Catalogue des textes liturgiques et des petits genres religieux», Grundriss der romanischen Literaturen des Mittelalters, Heidelberg, Winter, VI/2, 1970, pp. 19-53.

(28) $\$ 11,732-776$, pp. 39a-40a.

(29) Voir supra, n. 21. Cet ouvrage est désormais inséparable de son complément: K. V. SincLaIR, Prières en ancien français. Nouvelles références, renseignements complémentaires, indications biblio- graphiques, corrections et tables des articles du "Répertoire" de Sonet, Hamden, Connecticut, Archon Books, 1978.

(30) Ainsi par exemple 320, 677 et 1352.

(31) Ainsi 30 (par Jean Meschinot) ou 159 (de Jean Regnier).

(32) French devotional texts of the Middle Ages, A Bibliographical Manuscript Guide, compiled by K. V. Sinclair, Wesport, Connecticut-London, Greenwood Press, 1979.

(33) 2520

(34) 93.

(35) Voir supra, n. 21 et 29. 
qu'employait Deschamps travaillant à ce genre ${ }^{36}$, ce qui n'est pas tout à fait exact: Deschamps compose bel et bien un chant royal en onzains rimant (sur cinq timbres) comme la présente pièce: ababccddedE, cependant (différence secondaire) avec envoi sixain rimant ddeddE (en manière de deux tercets enchaînés) et (différence majeure) en vers coupés (dix des onze vers du couplet sont des décasyllabes, le cinquième est un heptasyllabe) $)^{37}$. Du reste, dans son Art de dictier, le même Eustache Deschamps donne de l'envoi des chançons royaulx une définition qui s'ajuste exactement à celui de la pièce qu'on vient d'évoquer ${ }^{38}$.

Quoi qu'il en soit, le texte issu du manuscrit de Jean Miélot, qu'on peut par conséquent dater, vraisemblablement, du deuxième tiers du $\mathrm{Xv}^{\mathrm{e}}$ siècle, correspond (d'ailleurs dans le droit fil de cet exemple formel de Deschamps, hétérométrie du couplet mise à part) à la définition palinodique du chant royal à l'époque ${ }^{39}$. On trouve bien, dans les archives du Puy de Rouen, un chant royal de cette sorte, attribué à Nicole Osmont, paraphrasant justement l'Ave Maria ${ }^{40}$. Le poète le consacre - Puy rouennais oblige - à la défense et illustration de la Conception immaculée; si l'on part du refrain - «De ung fils tout beau la mere toute belle» - on voit bien comment la Salutation angélique et l'Incarnation impliquent le motif - initial et primordial, suivant le fondement théologique de l'Institution normande - de la Tota pulchra ${ }^{41}$. Ajoutons que les cinq onzains se présentent séparément comme la paraphrase argumentée des propositions successives de l'Ave Maria: I. Ave Maria / II. Gratia plena / III. Dominus tecum / IV. Benedicta tu in mulieribus / V. Et benedictus fructus ventris tui. La méthode est donc proche de celle du poème attribuable à Jean Miélot (mais plus proche encore des paraphrases de l'Ave Maria dans les poèmes strophiques).

Cependant, le Puy rouennais de la Conception remonte à 1486. Ce poème de Nicole Osmont relève du XVI ${ }^{\mathrm{e}}$ siècle ${ }^{42}$ : il entreprend, d'une manière qui n'est peutêtre pas inédite, de paraphraser dans la forme du chant royal la prière commune, mais trop tardivement pour apporter quoi que ce soit à l'explication génétique du poème hébergé par le manuscrit de Jean Miélot. L'auteur d'«Ave fu dit», probablement, inaugure la paraphrase de l'Ave Maria sous forme de chant royal. Le fait-il en fonction des circonstances? Étant donné qu'il ne nous est pas parvenu, semble-t-il, d'autre exemple comparable de cette forme prenant pour objet cette prière, on ne saurait se prononcer.

(36) Art. cité, p. 219: «Cette structure [celle du chant royal à éditer] ne se retrouve pas parmi les types pourtant très variés des "Chansons royales" d'Eustache Deschamps».

(37) Voir Euvres complètes d'Eustache Deschamps, publiées d'après le manuscrit de la Bibliothèque nationale par le marquis de Queux de Saint-Hilaire (tomes I à VI) et par Gaston Raynaud (tomes VII à XI), Paris, 1878-1903 (S.A.T.F.), XI, p. 124 (avant-dernier exemple). Il s'agit de la pièce 403 du poète.

(38) Éd. citée, VII, p. 278 (nous citons Deschamps en donnant entre crochets des précisions qui visent à l'intelligence du texte): «Et doivent les envois d'icelles chançons, qui se commencent par Princes, estre de cinq vers entez par eulx aux rimes de la chançon sanz rebrique [= "refrain"]; c'est assavoir .II. vers premiers [dd], et puis un pareil de la rebriche [= "pareil au timbre de rime du refrain", soit, en effet, e]; et les .II. autres suyans les premiers [dd], deux concluans en substance l'effect de ladicte chançon et servens a la rebriche [E terminal]...».

(39) Voir par exemple G. Gros, Le Poème du Puy marial. Étude sur le serventois et le chant royal du XIV siècle à la Renaissance, Paris, Éditions Klincksieck, 1996, pp. 129-140.

(40) Voir D. Hüe, La Poésie palinodique à Rouen (1486-1550), Paris, Honoré Champion, 2002 (Bibliothèque Littéraire de la Renaissance, Série 3, tome XLIV), pp. 589-596, et spécialement pp. 591-593.

(41) Voici justement l'Argument dont le quatrain précède le texte de ce chant royal (d'après D. HüE, op. cit., p. 591): «Chant royal de devotion Qui la Vierge royne celique Prouve belle en sa conception, Par le beau salut angelique».

(42) Vraisemblablement entre 1510 et 1524; voir D. HüE, op. cit., p. 287. 
Voilà qui, malgré tout, n'empêche pas de se souvenir que le Puy d'Amiens, auquel a été présenté le chant royal qui suit celui-là dans le manuscrit de Jean Miélot, célébrait, par des poèmes, les fêtes mariales ${ }^{43}$ - dont, évidemment, l'Annonciation ${ }^{44} .$.

Durant toute leur carrière, ces Puys citadins de la France du Nord ont invité, ou attiré, des personnalités extérieures, de très haut rang parfois. Par exemple, le comte et trouvère Thibaut de Champagne n'avait-il pas en son temps participé au Puy d'Arras? ${ }^{45}$

GÉRARD GROS

(43) Voir G. Gros, Le Poète, la Vierge et le prince du Puy. Etude sur les Puys marials de la France du Nord du XIV siècle à la Renaissance, Paris, Editions Klincksieck, 1992, (Sapience), pp. 54-55.

(44) Un indice (à défaut de preuve) en faveur de la destination de ce chant royal à un Puy picard (et prioritairement à celui d'Amiens!) est fourni par plusieurs picardismes qu'on trouve dans ses vers, affectant souvent des mots placés à la rime: lignie, v. 7 (et 30); envoïe, v. 21; soullie (pour souillée), v. 41 et prisie, v. 56. Sur cette particularité phoné- tique (où joue aussi la pratique de l'analogie), voir par exemple C.T. Gossen, Grammaire de l'ancien picard, Paris, Éditions Klincksieck, 1970 (Bibliothèque française et romane, Série A: Manuels et Études linguistiques, 19), $\mathbb{\$} 8$, p. 55 (yod + ata $>$ pic. -ie (franc. -iée).

(45) Voir par exemple L. Selaf, 'Chanter plus baut'. La chanson religieuse vernaculaire au Moyen Âge (essai de contextualisation), Paris, Honoré Champion, 2008 (Nouvelle Bibliothèque du Moyen Âge, 87), p. 474. 


\section{Édition du texte ${ }^{46}$}

Ave fu dit pour salutacion

Que Grace I Dieu, doulce et contemplative,

Tramist I ça jus pour l'incarnation

De Dieu le l filz, au pere placative ${ }^{47}$.

Car Sapïence ou son II secret estoit

De remede remedier vouloit

Et I pourveoir a l'umaine lignie

Qui par pechié I estoit morte et perie.

Mais haultement fu de I ce relevee

Quant la Vierge glorieuse, Marie I,

Par Gabrïel fu de Dieu saluee.

Marïa fu sainctisme et propre nom

A la I dame, voye preparative

En laquelle I nostre salvation

Et nostre grace estoit expecltative ${ }^{48}$.

Pour quoy? Pour ce qu'en elle demourol[it]

Humilité, que Jhesus moult amoit,

Virginilté par concorde assoufie ${ }^{49}$

Et vray amour I, dont elle estoit remplie

A la tres digne et I notable journee

Que d'une voix par les cieulx I envoïe

Par Gabrïel fu de Dieu saluee.

Gratia vint de grant provisïon

Pour relparer l'amende primitive

Et aux hulmains baillier redemption,

Plaisir, liesse et $\mid$ joye infinitive ${ }^{50}$ :

Grace, pour vray tres bien I dire on le doit,

Car, se Rigueur eust tenu a I l'estroit

La grief paine que Adam ot deservie I,

Encores fust sa lignie asservie

Comme elle estoit lors que la bieneuree,

Pour acomplir | la saincte prophecie,

Par Gabrïl fu de Dieu saluee.

Plena estoit de benediction

Ceste dame, de I biens suppellative ${ }^{51}$,

Car en sa char prist I sa conjonction

(46) Restituant la disposition versifiée du texte, nous indiquons par la barre oblique la fin de ligne et, deux fois, par le même signe redoublé, le changement de page. Nous sommes responsable de la ponctuation et des majuscules de déférence (par exemple Dieu, vv. 2, 4, 37, 46, 56 et au refrain; Seigneur, vv. 39 et 47; Createur, v. 48; Saint Esperit, v. 42; Vierge, vv. 10, 41; Gabriel au refrain; Adam, v. 29), ou de personnification (Grace Dieu, v. 2, Sapience, v. 5 et Rigueur, v. 28). Nous résolvons les abréviations relevant en grande partie de la nasalisation des voyelles (par exemple bieneuree, v. 31, acomplir, v. 32, benediction, v. 34, comme, v. 40, Dominus et domination, v. 45), mais aussi deservie, v. 29, que, v. 31, pour, v. 32, quant, v. 38. Nous donnons une version désagglutinée par exemple de Ala, vv. 13 et 20, tresdigne, v. 20, lamende, v. 24, tresbien, v. 27, lestroit, v. 28, lancelle, v. 39 et aveoir v. 58.

$(47)=$ "propre à apaiser".

(48) = "d'un effet attendu".

(49) $=$ "pourvue".

$(50)=$ "infinie".

(51) = "précellente". 
Le fil de Dieu par maniere I optative ${ }^{52}$,

Quant elle dist a cil qui saluoit I:

«Vecy l'ancelle a mon Seigneur, fait soit

$\mathrm{Ta}$ | parole comme je l'ay ouye».

En ce disant la Vierge non soullie

Du I Saint Esperit conchu noble portee,

Aprés ce I que sa personne anoblie

Par Gabriel fu de I Dieu saluee.

Dominus est, et domination

Affiert $a^{53}$ Dieu I, sur tous imperative ${ }^{54}$,

Qui est Seigneur I devant creation

Et Createur de l'ame sensiltive

Et de tout ce que nommer on porroit I,

Pere a fille que creé il avoit,

Fil a mere, qui I souëf l'a nourrie

Et qui depuis a esté collolquie ${ }^{55}$

Roÿne es cieulz, lez son fil couronnee I,

Laquelle, estant en ceste mortel vie,

Par Gabrïel I fu de Dieu saluee.

Tecum fu Dieu, sainte dame prisie I,

Pour tes servans advocate et amie I,

De tout bon cuer a veoir desiree

Et celle qui I, pour nostre courtoisie ${ }^{56}$,

Par Gabrïel fu de Dieu I saluee.

Dans la présente liste des variantes et des notes, nous tenons compte de la version du manuscrit de Bruxelles, que nous désignons sous le sigle $\mathrm{B}$; nous appliquons le sigle $\mathrm{P}$ au manuscrit de Paris. - 14: $\mathrm{n}$. salvation $\mathrm{B}$; $\mathrm{n}$. salutation $\mathrm{P}$, rime, dans ce dernier, du même au même avec le v. 1 (bévue graphique, avec le premier t en trop); nous corrigeons $\mathrm{P}$ grâce à la leçon de B. - 16: les deux dernières lettres du vers absorbées dans le pli de la reliure; nous les restituons. - 18: par B; pas $\mathrm{P}$; nous corrigeons $\mathrm{P}$ grâce à la leçon de $\mathrm{B}$. - 26: vers omis dans $\mathrm{B}$, comme dans l'édition de Louis Mourin; Arthur Längfors l'avait signalé (art. cité, p. 165; voir supra, n. 23); $\mathrm{P}$ permet de restituer le libellé de ce vers. - 27: dir o. P; nous corrigeons, en accord avec $\mathrm{B}$. - 30: vers omis dans $\mathrm{P}$; nous le rétablissons à partir de $\mathrm{B}$, où il figure à cette place, et d'autre part grâce à $\mathrm{P}$ lui-même, qui copie ces vers 29 et 30 au cour du quatrième couplet; la 1. P; sa 1. B; nous amendons la leçon de $\mathrm{P}$ en recourant à celle de $\mathrm{B}$. - 41-42: La grief paine que | Adam ot deservie, Encores fust la lignie as $\left[18^{\mathrm{r}}\right]$ servie $\mathrm{P}$, qui, erronément, répète ici le vers 29, et restitue le vers 30 omis. - 39: f. soy B; Arthur Langfors avait ici aussi remarqué l'anomalie (ibid.): «Le texte paraît correct, seul le v. IV 39 inspire des doutes (...). La rime demande soit; mais il faudrait le féminin faite, qui cependant ferait un vers trop long»; P fournit le mot-rime exact. - 46: hésitation de $\mathrm{P}$ dans la graphie de sur (la troisième lettre ressemble à f): un choix difficile entre sur et sus?; *impative P: le trait d'abréviation, sur la bampe de p, est par erreur omis. - 49: nomme (à la rigueur nommé) o. p. P; nous corrigeons, conformément à B. - 50: nourrie B et P; la syntaxe exigerait nourri (cf., supra, le problème au vers 39). - 53: 1. ton $\mathrm{f}$. B. - 56: Tecum fu de D. P; nous corrigeons ici le texte, qui fait contresens (et donne un vers bypermétrique), grâce à la leçon de B: Tecum fu Dieu, dame sainte, prisie.

$(52)=$ "de souhait".

(53) $=$ "Appartient à".

(54) = "susceptible d'ordonner".

(55) = "établie".

(56) = "pour nous favoriser". 